\title{
Particle Swarm Optimization-Based Direct Inverse Control for Controlling the Power Level of the Indonesian Multipurpose Reactor
}

\author{
Yoyok Dwi Setyo Pambudi, ${ }^{1,2}$ Wahidin Wahab, ${ }^{1}$ and Benyamin Kusumoputro ${ }^{1}$ \\ ${ }^{1}$ Department of Electrical Engineering, University of Indonesia, Kampus Baru UI, Depok 16424, Indonesia \\ ${ }^{2}$ Center for Nuclear Reactor Technology and Safety, National Nuclear Energy Agency of Indonesia (BATAN), \\ Puspiptek Area, Serpong, Tangerang Selatan 15310, Indonesia
}

Correspondence should be addressed to Yoyok Dwi Setyo Pambudi; yoyok.pambudi@yahoo.com

Received 18 November 2015; Revised 5 May 2016; Accepted 8 May 2016

Academic Editor: Eugenijus Ušpuras

Copyright (C) 2016 Yoyok Dwi Setyo Pambudi et al. This is an open access article distributed under the Creative Commons Attribution License, which permits unrestricted use, distribution, and reproduction in any medium, provided the original work is properly cited.

\begin{abstract}
A neural network-direct inverse control (NN-DIC) has been simulated to automatically control the power level of nuclear reactors. This method has been tested on an Indonesian pool type multipurpose reactor, namely, Reaktor Serba Guna-GA Siwabessy (RSGGAS). The result confirmed that this method still cannot minimize errors and shorten the learning process time. A new method is therefore needed which will improve the performance of the DIC. The objective of this study is to develop a particle swarm optimization-based direct inverse control (PSO-DIC) to overcome the weaknesses of the NN-DIC. In the proposed PSO-DIC, the PSO algorithm is integrated into the DIC technique to train the weights of the DIC controller. This integration is able to accelerate the learning process. To improve the performance of the system identification, a backpropagation (BP) algorithm is introduced into the PSO algorithm. To show the feasibility and effectiveness of this proposed PSO-DIC technique, a case study on power level control of RSG-GAS is performed. The simulation results confirm that the PSO-DIC has better performance than NN-DIC. The new developed PSO-DIC has smaller steady-state error and less overshoot and oscillation.
\end{abstract}

\section{Introduction}

Controlling the research reactor power level is very complex and difficult due to the complexity and the nonlinearity of the dynamic behavior of nuclear reactors. The reactor dynamic changes according to the power levels, ageing effects, and reactivity. Challenges in research reactor control are on how to increase or decrease the power from a certain level to a desired level and, at the same time, stable and safe condition are still maintained $[1,2]$. The reactor power level control system can be managed either manually by an expert operator or automatically by conventional controllers, such as a Proportional Integral and Derivative (PID) [3, 4]. However, both these control mechanisms suffer from performance degradation and lack of safety [5]. Another type of controllers, which has been applied to control power reactor, is $H_{\infty}$ optimal controller [6]. This controller has better performance than classical controllers, but its order is still large. Neural network technique was then introduced by a number of researchers to develop a reliable and simple controller system for a research reactor. We encounter from literatures that there have been a few studies on the applications of artificial neural networks (ANNs) to control the power of nuclear reactors $[4,7]$. An artificial neural network is a system, which consists of interconnected neurons to memorize the knowledge of the plant. As a controller, neural networks can mimic the scientific model of nonlinear systems and their inverse. Typically, neural network systems are initially fed up with amounts of data to train them finding the correlation pattern between inputs and outputs. The popular model of a multilayer neural network is backpropagation neural networks (BPNN), which usually consists of three layers, 
that is, an input layer, an output layer, and a hidden layer. However, BPNN still has disadvantages, such as lack of ability to find global optimistic result and easily falling into a local minimum, which slows down the learning process.

In order to improve the BPNN learning mechanism, particle swarm optimization (PSO) algorithm was utilized [8]. The PSO algorithm has a strong ability to generate weight of nonlinear systems modeled in neural network. This PSO algorithm has been implemented in nuclear area to identify nuclear power plant transients $[9,10]$, to optimize pressurized water reactor's fuel loading [11-13], and to identify reactor components [14].

In the meantime, neural network-direct inverse control (NN-DIC) has been simulated to automatically control the power level of an Indonesian pool type multipurpose reactor, namely, Reaktor Serba Guna-GA Siwabessy (RSG-GAS). Basically, NN-DIC method is usually based on backpropagation learning algorithm. Unfortunately, this method still cannot minimize errors and shorten the learning process time. Therefore, it is necessary to develop a new method, which can improve the performance of the NN-DIC to control the power level of RSG-GAS.

The objective of this study is to develop a particle swarm optimization-based direct inverse control (PSO-DIC) to overcome the weaknesses of the NN-DIC. Due to its strong ability to find the global result of nonlinear systems using a heuristic process, the PSO algorithm will accelerate the learning process of the DIC controller to find its weight. To improve the performance of the system identification, a backpropagation (BP) algorithm is then introduced into the PSO algorithm. To show the feasibility and effectiveness of this proposed PSO-DIC technique, a case study on power level control of RSG-GAS is performed. The PSO-DIC controller is implemented at various power levels.

The paper is organized as follows. Section 2 briefly describes the Indonesian pool type multipurpose reactor: Reaktor Serba Guna-GA Siwabessy (RSG-GAS). The PSODIC method is described in Section 3. Section 4 discusses the results of the simulations to show the feasibility and effectiveness of the PSO-DIC. Finally, the conclusions are given in Section 5.

\section{Reaktor Serba Guna-GA Siwabessy (RSG-GAS)}

Reaktor Serba Guna-GA Siwabessy (RSG-GAS) is a multipurpose reactor which is operated by the National Nuclear Energy Agency of Indonesia (BATAN) [15]. It is a light watercooled and moderated pool type reactor. The primary purposes of this reactor are for research and isotope productions. The nominal power is $30 \mathrm{MW}$ and produces thermal neutron flux in the order of $10^{14} \mathrm{n} / \mathrm{cm}^{2}$ s. The reactor uses $\mathrm{U}_{3} \mathrm{Si}_{2} \mathrm{Al}$ MTR-type fuel element with $19.75 \%{ }^{235} \mathrm{U}$ enrichment. In the beginning of cycle (BOC), the total excess reactivity of RSGGAS fuel rod is $9.7 \%$. The reactor core contains 40 fuel assemblies and 8 control rod assemblies, consisting of seven bank rods, which can move together and be assembled to function as a safety rod, and 1 regulator rod. The regulator rod is made of materials, which have high burn-up ratio. Meanwhile, the bank rod is made of materials, which have low burn-up ratio. Therefore, the regulator rod will be able to compensate power changes and to maneuver reactor power level. Total reactivity value of those 8 control rods is $-13.8 \%$.

The schematic diagram of coolant flow and sensor position of RSG-GAS is shown in Figure 1.

In the reactor core, there are four neutron flux detectors, which are located at four different positions. Those detectors are designed redundantly by 2 -out-of- 4 . This redundancy means the signal from at least 2 sensors must exist in order to ensure the plant's safety and availability [15].

The power level of RSG-GAS is controlled by withdrawing or inserting control rods from or to the reactor core. The control rod movement is adjusted by a servo motor with a maximum speed of $0.0564 \mathrm{~cm} / \mathrm{s}$. Withdrawing the control rod from the reactor core will raise neutron production. As the neutron flux rises, the thermal power of the reactor will also increase. The control input of reactor power is the position of the regulator rod and the position of the bank rod. A block diagram of the power level control system for RSGGAS reactor is shown in Figure 2.

Reactor power level is measured by boron-coated ionization chambers (BCIC) detector, which has neutron sensitivity of approximately $5 \times 10^{-14} \mathrm{~A} / \mathrm{nV}$. The $\mathrm{DC}$ signal of this detector is transmitted and amplified by a linear DC amplifier and is converted into a standard signal of 0 to $10 \mathrm{~V}$. The data from reactor sensor is sampled and controlled by Siemens S7 300 DAQ with sampling time of $2 \mathrm{~Hz}$. For monitoring purposes, the data is transmitted to the monitoring computer located in the reactor control room.

Currently, control system of RSG-GAS has been managed by two modes, that is, manually and automatically. Manual control is performed by an expert operator and automatic control is done by a PID controller, but only for one control rod at steady-state condition. The disadvantage of manual control is that it can get affected by the presence, mood, and experience of the operator. Automatic control in RSG-GAS works in close loop by comparing setpoint value to an output power. Automatic mode with this PID has the disadvantage that it only works to maintain the reactor at steady-state position. Meanwhile, manual control is still used for startup and maneuver power level of RSG-GAS reactor. This is necessary for applied automatic power control system capable of controlling the power from a start-up until it reaches operating power reactor. The operating power level of RSGGAS is $15 \mathrm{MW}$.

To achieve a $15 \mathrm{MW}$ reactor power level, the RSG-GAS operator has arranged the scenario to gradually increase the power reactor level from $2 \mathrm{MW}, 5 \mathrm{MW}, 10 \mathrm{MW}$, and $15 \mathrm{MW}$ as shown in Figure 3. The purpose of this scenario is to keep the reactor core stable and to prevent reactor from scram (automatic shutdown of the reactor). In addition, this scenario will enable operators to monitor the condition of the reactor safety parameters in each step. A total of 9000 data samples are taken which describes the inputs and outputs of start-up manual operation of RSG-GAS reactor. The data sets are displayed in Figure 3. 


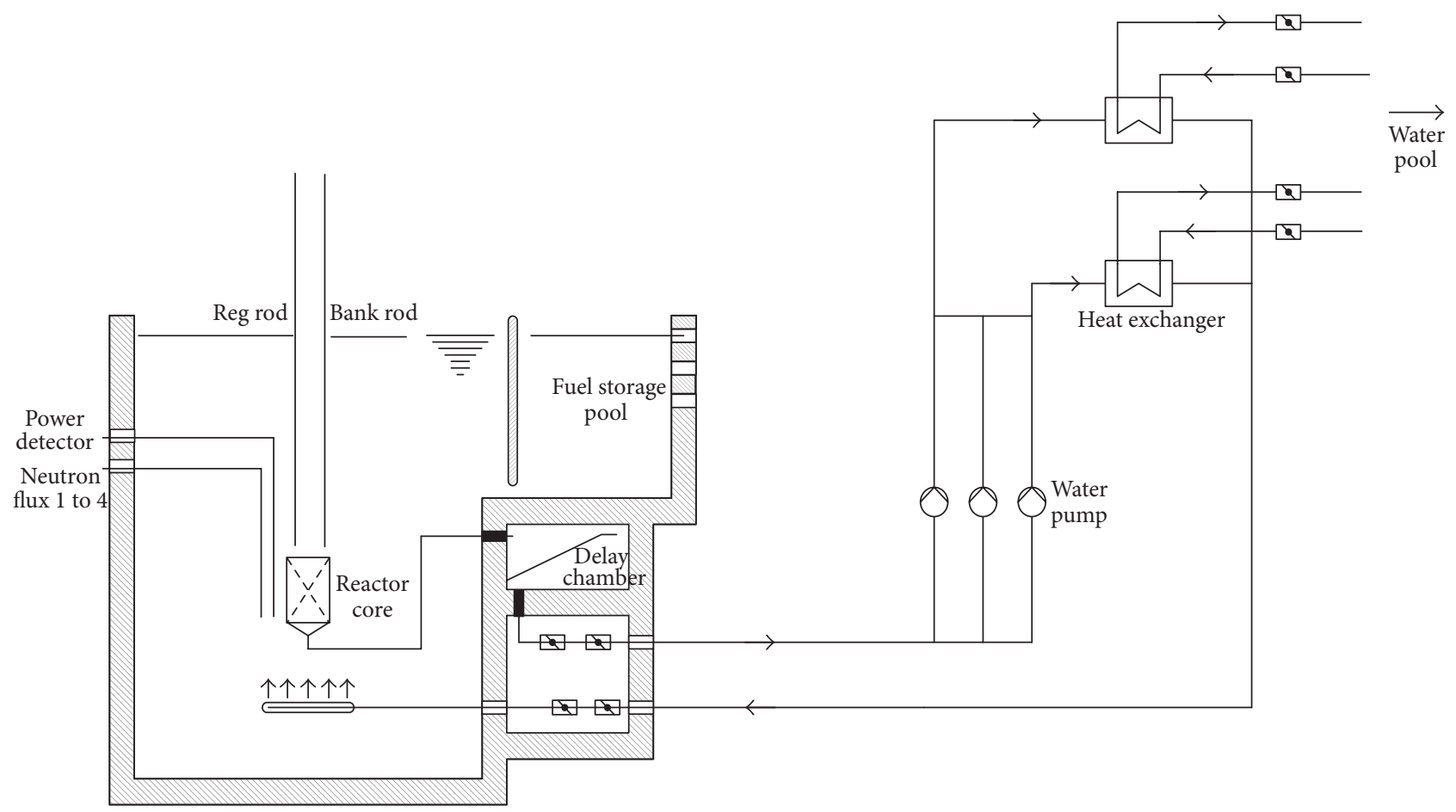

FIGURE 1: Coolant flow and position sensors in RSG-GAS [15].

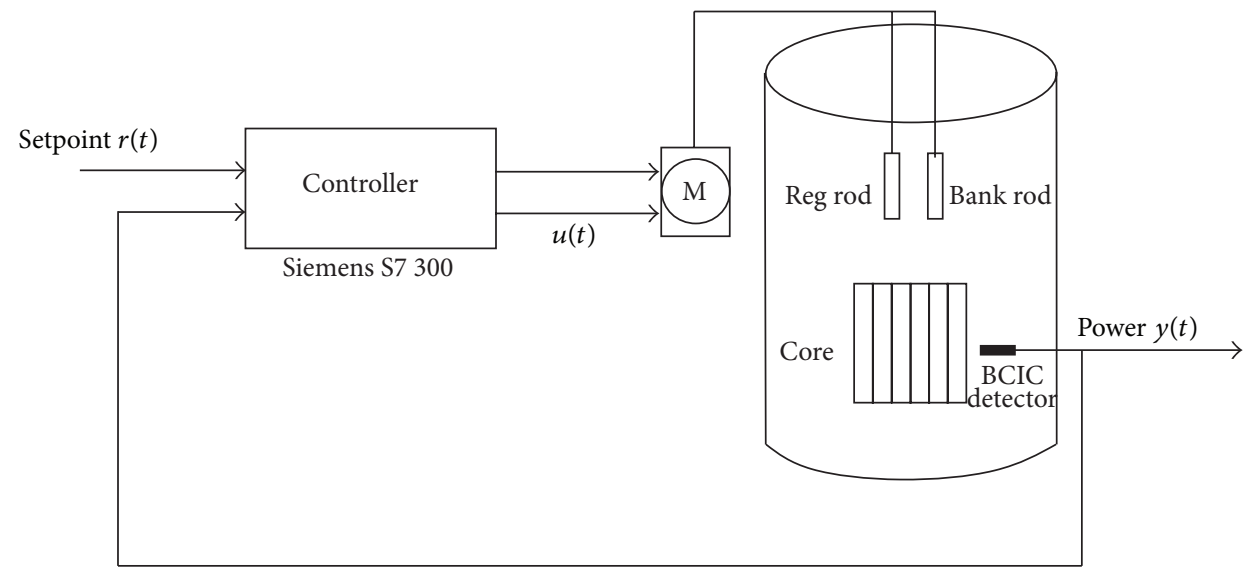

FIGURE 2: Block diagram of the RSG-GAS power level control.

\section{Particle Swarm Optimization-Based Direct Inverse Control and PSO-BP Algorithm}

In this section, PSO-DIC for power level control of RSG-GAS reactor and the proposed PSO-BP algorithm for RSG-GAS identification are described.

3.1. Particle Swarm Optimization-Based Direct Inverse Control. Direct inverse control (DIC) basically is the control of nonlinear plants by training the neural network part to act as an inverse control of the plant to cancel its dynamics. The inverse controllers were cascaded with reactor models. The cascaded system then gave an identity mapping between setpoint signals and controlled plant responses. The neural network that was used as the controller is a multilayer perceptron with one hidden layer and the learning mechanism is accomplished by using a PSO method. Learning is done by adjusting the connections weight between neurons in the hidden layer and output layer based on a set of input and output patterns of the plant. During the iterative process, PSO training was used by exploiting an error based fitness function for this process. An error is obtained from the difference between reference signal and output signal of the control system. The training procedure continues until the network output provided a Mean Square Error (MSE) less than a determined value.

The generalized architecture of DIC of RSG-GAS reactor is shown in Figure 4. The block diagram shows that the DIC 

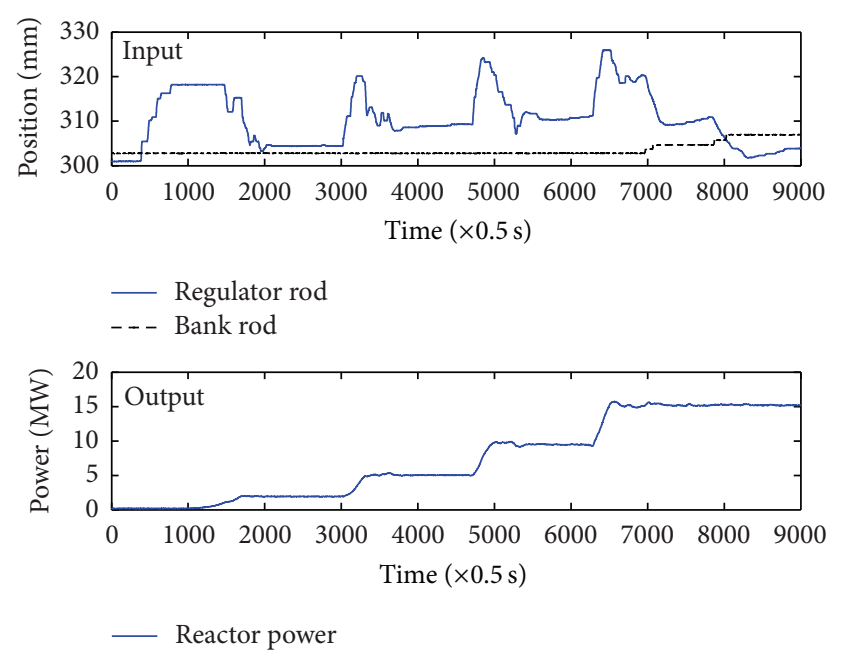

FIGURE 3: Input and output data of RGS-GAS experiment.

can be divided into two parts, namely, (1) inverse controller and (2) reactor model. The reference signal $r(t)$ of DIC is the power level setpoint of the reactor and the output of inverse controller is the position of the control rod, that is, the regulator rod position $u_{1}(t)$ and bank rod position $u_{2}(t)$. In the reactor model, the position of control rod acts as the input of RSG-GAS while the output is the power level reactor $y(t)$. The power level was used as feedback control to the desired setpoint. The inverse controller and system identification design in this paper were carried out on the MATLAB platform.

The block diagram of inverse controller of PSO-DIC was shown at Figure 5(a). The function of neural network representing inverse of the RSG-GAS system is given by [16]

$$
\begin{aligned}
& u(t)=f\left[u(t-1), u(t-2), \ldots, u\left(t-N_{x}\right), \ldots, y(t+1),\right. \\
& \left.y(t), \ldots, y\left(t-N_{y}\right)\right]
\end{aligned}
$$

where $y(t)$ is the output of the model, $u(t)$ is the input of the model, $N_{x}$ denotes the maximum lag of the input plant and $N_{y}$ is the maximum lag of the output, and $t$ is time.

This inverse network model consists of 10 input neurons, 20 hidden neurons, and 2 output neurons. The neural network architecture is determined by using input neuron that consists of the input and output signals with $N_{x}=3$ and $N_{y}=3$, respectively.

The input $u(t-1), u(t-2), \ldots, u\left(t-N_{x}\right)$ and $y(t+$ 1), $y(t), \ldots, y\left(t-N_{y}\right)$ are multiplied by weights $v_{u n p}$ and $v_{y n p}$, respectively, and summed at each hidden neuron. The sigmoid activation function is applied to this summed value yielding the output of the hidden neuron. After multiplying the hidden neuron output by weights $w_{p}$, then the output $y(t)$ can be calculated as

$$
y(t)=\sum_{p=1}^{P} w_{p}\left(\frac{1}{1+e^{-\left(\sum_{1}^{N_{x}} u\left(t-n_{x}\right) v_{u n p}+\sum_{1}^{N_{y}} y\left(t-n_{y}\right) v_{y n p}\right)}}\right),
$$

where $n=1,2, \ldots, N$ is the number of input neurons and $p=$ $1,2, \ldots, P$ the number of the hidden neurons. The difference value between output of the plant $y(t)$ and the output of the inverse model $\hat{y}(t)$ is calculated using minimization function Mean Square Error as follows:

$$
\operatorname{MSE}=\frac{1}{N} \sum 0.5(y(t)-\widehat{y}(t))^{2},
$$

where $N$ is the number of data.

During the training process, the PSO was used to obtain connection weight of inverse system. The training process was continued until a predefined termination criterion was fulfilled. The goal of the PSO-DIC is to get better results than the original DIC, such as the smaller Mean Square Error (MSE), settling time, and minimum overshoot.

In order to train NARX neural network for system identification and system inverse, the data set from RSG-GAS has to be normalized. Normalization implies that all values from data set should take values in the range from -1 to 1 . Formula for normalization is computed as follows:

$$
x_{i,-1 \text { to } 1}=2\left(\frac{x_{i}-x_{\min }}{x_{\max }-x_{\min }}\right)-1,
$$

where $x_{i}$ is each data point, $x_{\min }$ is the lower bound of data operation, and $x_{\max }$ is the upper bound of data operation.

Instead of using mathematical models, modeling of nuclear reactor system using neural network offers two advantages. It can generate results faster than other techniques' based model [17]. Moreover, it does not need to understand the dynamic of the plant due to its capability to manage nonlinear system. For the system identification of RSG-GAS reactor, neural networks combined with backpropagation and PSO algorithm are utilized through an identification learning scheme as depicted in Figure 5(b). Identification block contains an input layer with 11 input neurons, a hidden layer with 22 neurons, and an output layer with 1 output neuron. The architecture of NN was determined by using input neuron that consists of input signal of control rod position $u_{1}(t)$, input signal bank rod position $u_{2}(t)$, and output signal of power level reactor $y(t)$. The neural network is arranged using a Nonlinear AutoRegressive with eXogenous (NARX) input model structure, by feeding back the outputs to the input of the network. In nuclear area, NARX input model for identification of industrial furnace temperature of sintering process has been investigated effectively [18]. The model of NARX input can be derived from

$$
\begin{aligned}
& y(t)=f\left[y(t-1), y(t-2), \ldots, y\left(t-N_{y}\right), u(t),\right. \\
& \left.u(t-1), \ldots, u\left(t-N_{x}\right)\right],
\end{aligned}
$$

where $y(t)$ is the output of the model, $u(t)$ is the input of the model, $N_{x}$ denotes the maximum lag of the input plant, and $N_{y}$ is the maximum lag of the output with $N_{x}=3$ and $N_{y}=3$.

3.2. PSO-BP Algorithm for RSG-GAS Identification. The PSO algorithm is an optimization algorithm based on behavior population of birds or particles [19]. Any particles behave in a distributed manner by using its own intelligence and also influenced the behavior of the collective group. Typically, 


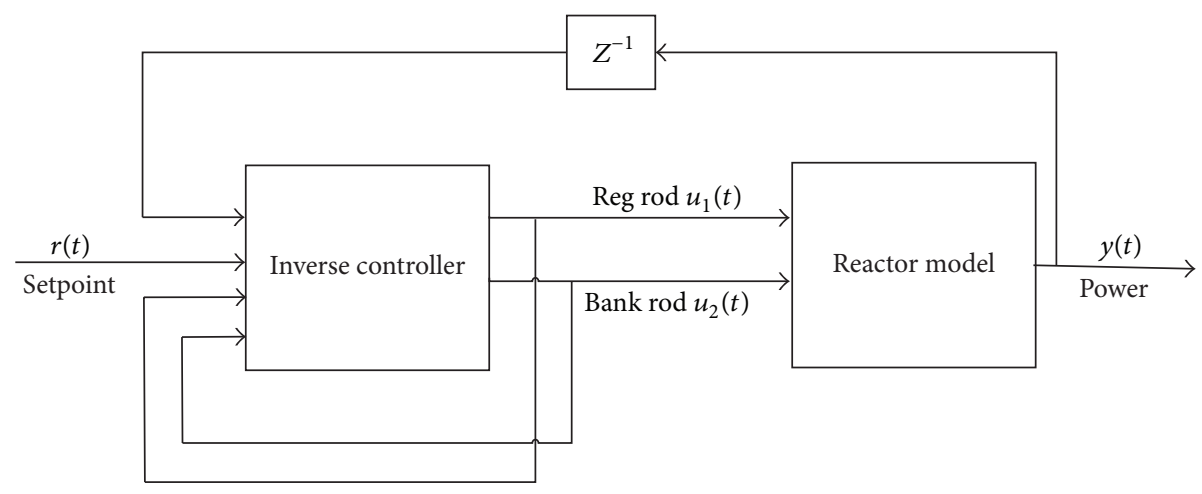

FIGURE 4: Block diagram of DIC on RSG-GAS.

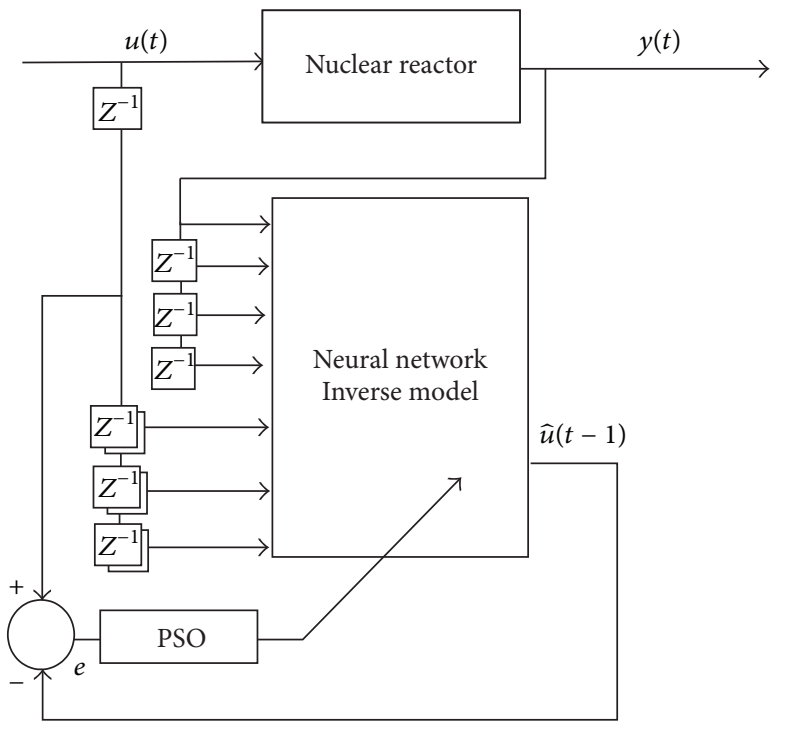

(a)

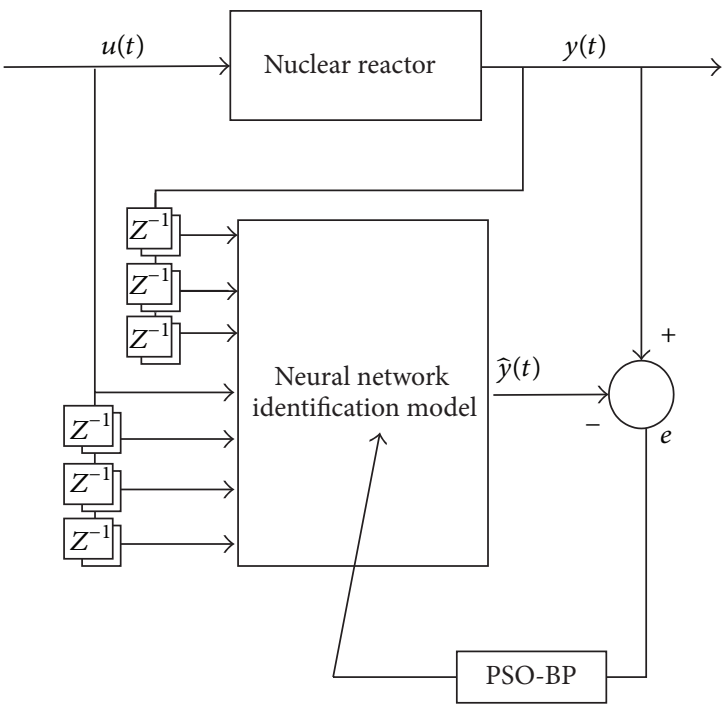

(b)

FIGURE 5: The block diagram of (a) inverse model training and (b) identification model training.

the algorithm uses two neighborhoods, that is, global neighborhood and local neighborhood. The local neighborhood topology has a greater possibility of finding a global optimum, which leads to this work, to use. After the improvement [20], the particle position and velocity update equations are represented as follows:

$$
\begin{aligned}
& V_{j}(i)= W \times V_{j}(i-1)+c_{1} \times r_{1} \times\left[P_{\text {best }}-X_{j}(i-1)\right] \\
&+c_{2} \times r_{2} \times\left[G_{\text {best }}-X_{j}(i-1)\right], \\
& X_{j}(i)= X_{j}(i-1)+V_{j}(i) \quad j=1,2,3, \ldots, N, \\
& W(i)=W_{\max }-\left(\frac{W_{\max }-W_{\min }}{i_{\max }}\right) i, \\
& c_{1}=c_{1 \max }-\left(\frac{c_{1 \max }-c_{1 \min }}{i_{\max }}\right) i, \\
& c_{2}= c_{1 \max }+c_{1 \max }-c_{1},
\end{aligned}
$$

where $W$ is the inertia weight, $V_{j}$ is the velocity of particle $i$ at iteration $j, c_{1}$ and $c_{2}$ are the positive values of acceleration coefficient, and $r_{1}$ and $r_{2}$ are random numbers between 0 and 1. $P_{\text {best }}$ is the best location of each particle; $G_{\text {best }}$ is the best location of neighbors. $X_{j}$ is the position of particle $i$. In this paper, every particle of PSO represents a set weight of feed forward neural network. For encoding strategy, every particle is encoded as a vector. For instance, particle for training the inverse system with 10 input, 20 hidden, and 2 output neurons was encoded as vector with $10 \times 20+20+20 \times 2$ +2 dimension.

The fitness function of the $i$ th training particle of PSO was defined as follows:

$$
\text { fitness }=\frac{1}{N} \sum \frac{1}{2}\left(y_{k}-\hat{y}_{k}\right)^{2},
$$

where $N$ is the number of data, $y_{k}$ is the desired output, and $\hat{y}_{k}$ is the actual output.

After predefined number of iterations has been reached, the PSO algorithm will get the optimum solution of weight 
of feedforward NN. In this paper, the initial value of particle was set in range $[-1,1]$ randomly. This interval is equal to the range of normalized data set of the plant. Table 1.

The parameter for optimized PSO-DIC was shown in

In order to have a better performance of identification system on producing a lower MSE output plant, optimization of identification system by combining PSO algorithm with BP was used. PSO algorithm is a global algorithm, with a heuristic process which has a strong ability to find the global result, but it has a disadvantage that the search around global optimum is slow. On the contrary, the BP algorithm has a strong ability to discover local optimistic results, but its ability to find the global optimistic result is weak [8]. Therefore, the $\mathrm{BP}$ algorithm is used to search the global optimum solution around its values.

The algorithm of hybrid PSO-BP for identification of RSG-GAS can be summarized into several steps as follows:

(1) Initialize maximum iteration, $c_{\max }, c_{\min }$, and minimum error MSE.

(2) Generate the position and velocity of a group of random particles (weight value for $\mathrm{NN}$ ) in the range of $[-1,1]$.

(3) Evaluate the fitness value (smallest MSE) of each particle and use $X_{j}$ as the current position of the particles, while $P_{\text {best }}$ is set as the best position of the initialized particles. Find $G_{\text {best }}$ as a global best position among initialized particles.

(4) Keep the best position of particles and then update the position and velocity of all particles using (6).

(5) Evaluate the fitness value of each new particle; then, replace the worst particle by the finest one.

(6) If the fitness value is not changed for 5 times, then switch to BP algorithm.

(7) Use the weight values obtained as weight of NN.

\section{Result and Discussions}

The experiments were conducted in RSG-GAS reactor by taking the data at the time of start-up operation and steady state of the reactor. The data from reactor sensor was sampled by Siemens S7 300 DAQ with sampling time of $2 \mathrm{~Hz}$ and transmitted to the monitoring $\mathrm{PC}$ in the control room. For validation of the proposed algorithm, some experiments for reactor power have been done by comparing the simulated results with manual operations by operators.

4.1. System Identification. In this research, the BP and hybrid PSO-BP are applied to the model of the RSG-GAS reactor. A sigmoid activation function for the output and hidden layer is used. The performance of the resulting identification system with a hybrid PSO-BP and BP was shown in Figure 6.

The identification of RSG-GAS reactor using back propagation for 212 epochs consumes CPU time of 127 seconds and gets MSE $7.739 \times 10^{-5}$. Meanwhile, the reactor identification plant using hybrid PSO-BP for 113 epochs (123 seconds) gets
TABle 1: Parameter for PSO-DIC.

\begin{tabular}{lcc}
\hline Parameter & Symbol & Value \\
\hline Iteration number & $i$ & 50 \\
Particle swarm size & $S$ & 30 \\
Number of trained parameters & $D$ & 262 (Ident) \\
& & 309 (Invers) \\
Acceleration coefficient (minimum) & $c_{1 \min }$ & 1.5 \\
Acceleration coefficient (maximum) & $c_{2 \max }$ & 2.0 \\
Minimum of inertia & $w_{\min }$ & 0.1 \\
Maximum of inertia & $w_{\max }$ & 0.9 \\
\hline
\end{tabular}

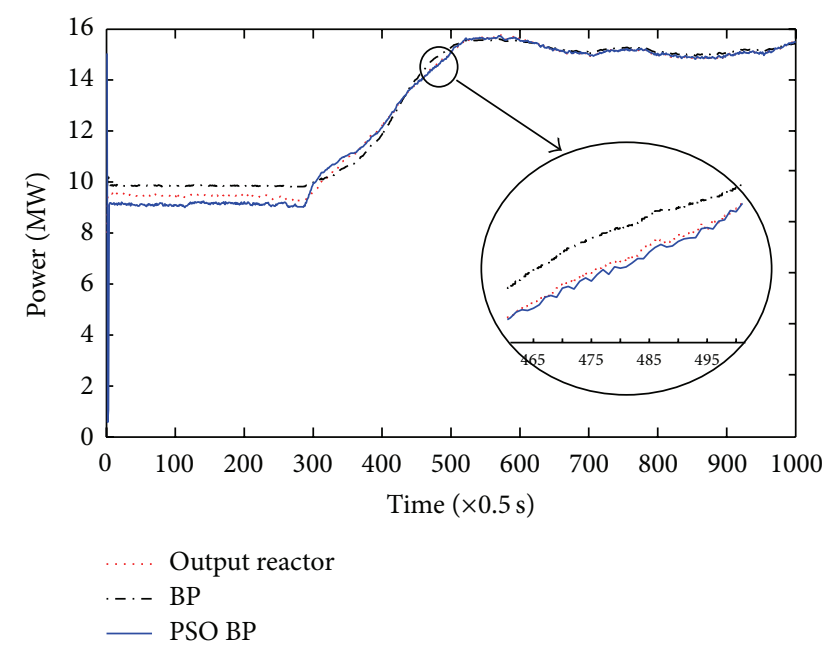

FIGURE 6: Comparison results of system identification of the RSGGAS using backpropagation and hybrid PSO-BP.

MSE $2.859 \times 10^{-5}$. Epoch is variable that represents iterative times. From these experiments, it can be concluded that, for the same goal, the PSO-BP algorithm using equivalent CPU time $(127 \times 123)$ to the $\mathrm{BP}$ algorithm will get higher training accuracy than the $\mathrm{BP}$ algorithm.

4.2. Direct Inverse Control (DIC) System. The development of RSG-GAS reactor control networks based direct inverse controller and its experiment for performance analysis are conducted as depicted in Figures $7(a)-7(d)$.

At first, it is asked to use the controller to control the reactor from an initial power of $200 \mathrm{~kW}$ to $2 \mathrm{MW}$. Simulation result of the RSG-GAS reactor control system is shown in Figure $7(\mathrm{a})$. The operator brought the reactor power from initial to desired power level. It shows that it needs settling time of about $275 \mathrm{~s}$ to achieve $2 \mathrm{MW}$. From the simulation, it showed that DIC could bring the reactor with the same trajectory of operation almost without an overshoot; the MSE was achieved at this state power level equal to $1.331 \times 10^{-4}$.

Secondly, the performance of the DIC that was used to control the reactor power from $2 \mathrm{MW}$ to $5 \mathrm{MW}$ was shown in Figure 7(b). At this stage, the DIC could bring the reactor to the desired power level in $107 \mathrm{~s}$ but with an oscillation and overshoot. The MSE was achieved as $1.11 \times 10^{-4}$. 


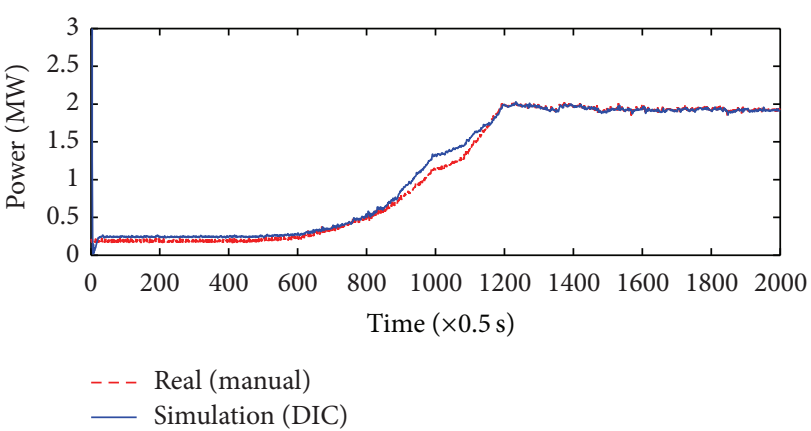

(a) $0.2 \mathrm{MW}$ to $2 \mathrm{MW}$

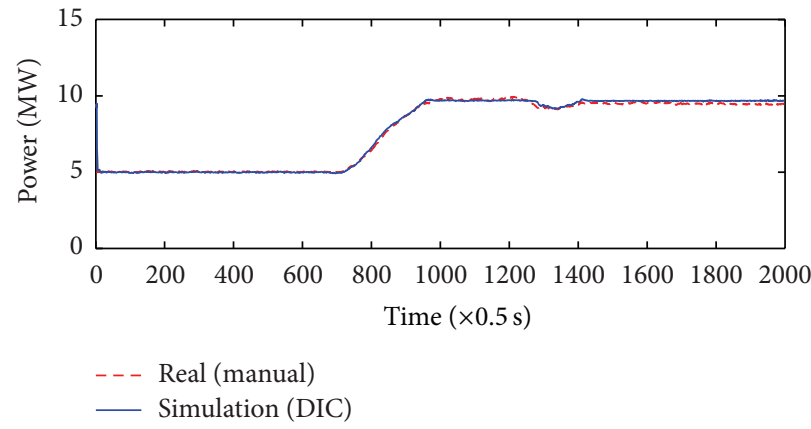

(c) $5 \mathrm{MW}$ to $10 \mathrm{MW}$

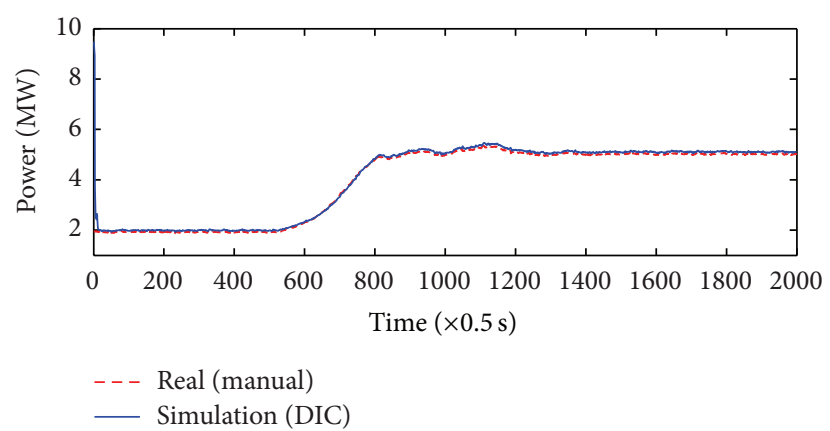

(b) $2 \mathrm{MW}$ to $5 \mathrm{MW}$

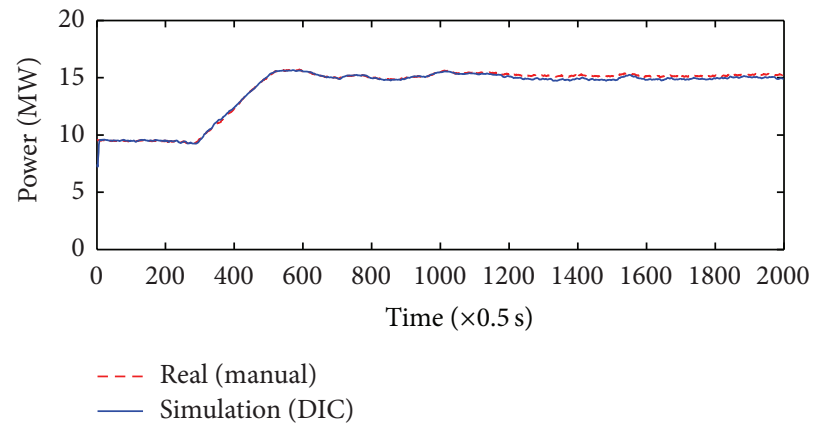

(d) $10 \mathrm{MW}$ to $15 \mathrm{MW}$

Figure 7: Response of using the DIC controller compared to manual control for the operating power at different power level.

Thirdly, the simulation test was used for power generation from initial power $5 \mathrm{MW}$ and final power was $10 \mathrm{MW}$ as shown in Figure 7(c). In this case, actually, the operator intended to manage power ascent to $10 \mathrm{MW}$, but the result of reactor power was $9.5 \mathrm{MW}$ and power reactor was oscillating. At this state, MSE was achieved as $1.434 \times 10^{-4}$.

Lastly, the RSG-GAS reactor was operated from the initial power $10 \mathrm{MW}$ to final power $15 \mathrm{MW}$ as shown in Figure 7(d). At this case, DIC could bring power to the desired power level in about $107 \mathrm{~s}$, but oscillating; overshoot was seen in this stage, with MSE of $8.753 \times 10^{-4}$.

The results show that the DIC was able to follow the trajectory given by the reactor's operator. However, the steady-state error and oscillated power will also occur as the DIC output, as the two components are also part of the trajectory. The power level oscillation will produce neutron flux oscillation in the reactor core.

4.3. DIC System with Specific Power Level Trajectory. In order to control the reactor's output power to the desired level, a specific trajectory should be given. This trajectory should be made by setting the desired setpoint (power level) exactly. After this, the retraining of inverse control was done by DIC and PSO-DIC to get the value of the corresponding weights.

The results of DIC and PSO-DIC were used to control power increase from 0.2 MW to $15 \mathrm{MW}$, as shown in Figures 8(a)-8(d). As seen from these figures, both the DIC and PSODIC could bring the reactor power to follow the setpoint trajectory with less overshoot and minimum MSE. When the power level reactor changed power level from $0.2 \mathrm{MW}$ to $2 \mathrm{MW}$, the steady-state error for the DIC controller is $0.42 \mathrm{MW}$, or $21 \%$, while the error for the PSO-based DIC is $0.05 \mathrm{MW}(2.5 \%)$ as depicted in Figure 8(a).

Meanwhile, Figure 8(c) shows that, to change the power level from $5 \mathrm{MW}$ to $10 \mathrm{MW}$, the steady-state error for the DIC controller is $0.28 \mathrm{MW}$, whereas that for the PSO-based DIC controller is $0,08 \mathrm{MW}$. This clearly shows that the performance of the PSO-based DIC controller is better than the original DIC controller. Moreover, it did not exhibit any oscillation. The overall comparison of DIC and PSO-DIC at RSG-GAS reactor is shown in Table 2. Based on controlling performance, PSO-based DIC is better than original DIC. It generates less steady-state error and less overshoot, without oscillation.

\section{Conclusion}

In this study, neural network using direct inverse and particle swarm optimization for identification and controlling the RSG-GAS reactor has been presented. A hybrid PSObackpropagation was used for identifying RSG-GAS nuclear reactor based on real data experiment. DIC was used to create inverse controller of the system. Improvement of the DIC was performed by using a PSO-DIC method, which significantly decreased the MSE of the controller and could bring the reactor power to desired power level relatively within a short time. This control system was tested for several reactor operating conditions. Simulation results confirmed that PSO-DIC controller can give satisfactory performance. 
TABLE 2: Comparison of DIC and PSO-DIC.

\begin{tabular}{|c|c|c|c|c|c|c|}
\hline \multirow{2}{*}{ Stage (MW) } & \multicolumn{2}{|c|}{ Settling time (s) } & \multicolumn{2}{|c|}{ Overshoot (MW) } & \multicolumn{2}{|c|}{$\operatorname{MSE}\left(\times 10^{-5}\right)$} \\
\hline & DIC & PSO-DIC & DIC & PSO-DIC & DIC & PSO-DIC \\
\hline $0.2-2$ & 200.35 & 135.83 & 0.034 & 0.0290 & 4.552 & 1.293 \\
\hline $2-5$ & 157.05 & 157.00 & 2.882 & 0.0434 & 5.869 & 4.503 \\
\hline $5-10$ & 204.97 & 155.10 & 1.035 & 0.0098 & 33.2 & 14.91 \\
\hline $10-15$ & 198.12 & 185.83 & 0 & 0 & 28.01 & 12.49 \\
\hline
\end{tabular}

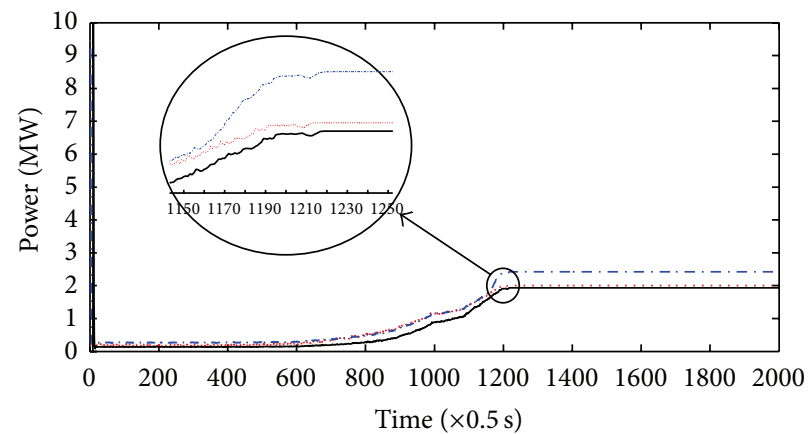

…. Setpoint

...- DIC

- PSO-based DIC

(a) $0.2 \mathrm{MW}$ to $2 \mathrm{MW}$

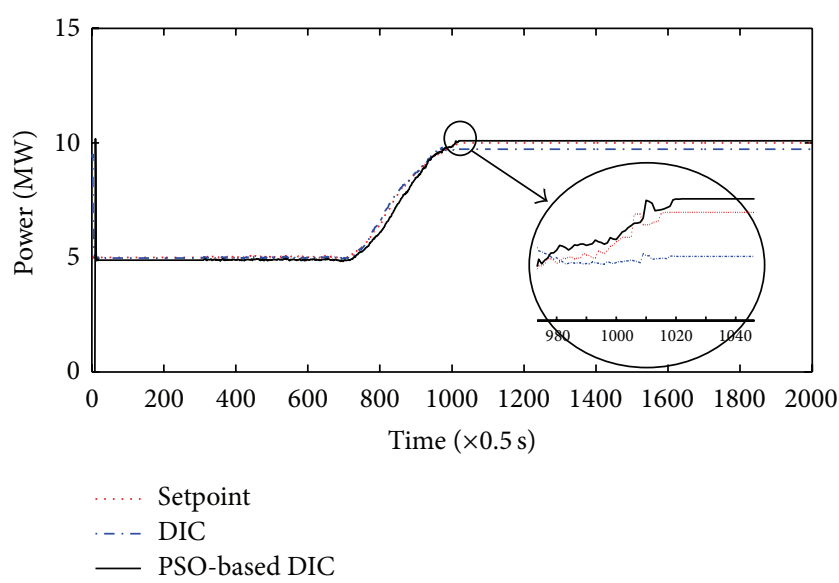

(c) $5 \mathrm{MW}$ to $10 \mathrm{MW}$

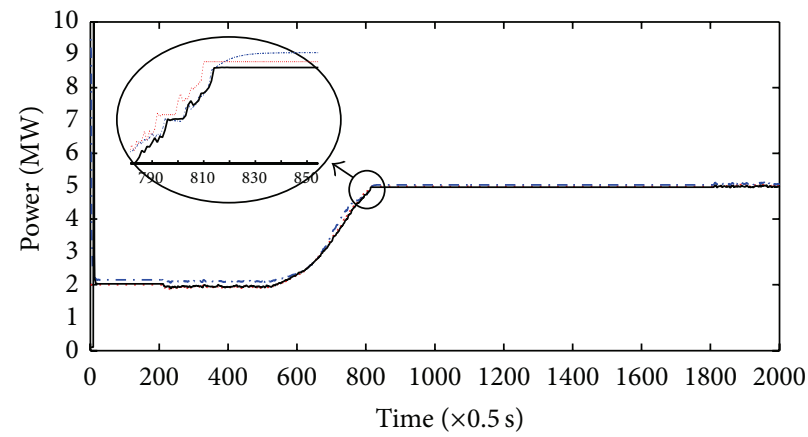

.... Setpoint

-..- DIC

— PSO-based DIC

(b) $2 \mathrm{MW}$ to $5 \mathrm{MW}$

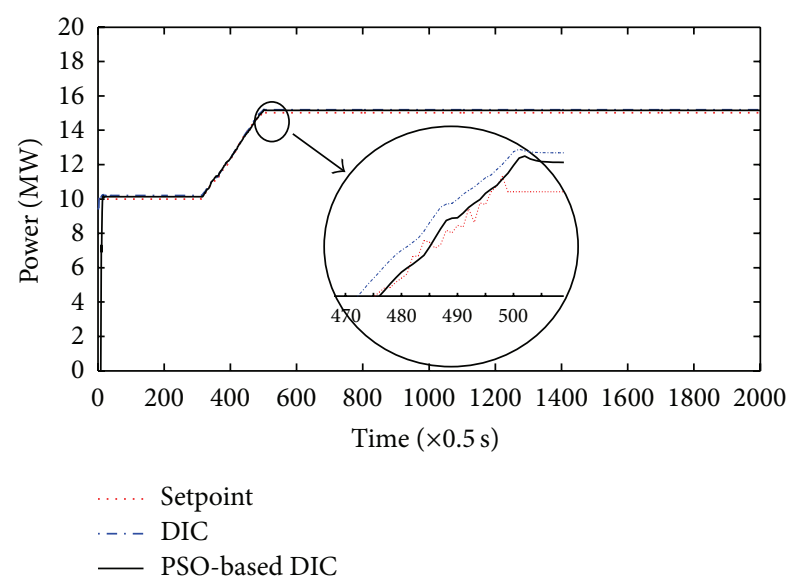

(d) $10 \mathrm{MW}$ to $15 \mathrm{MW}$

FIGURE 8: Comparison response of the DIC and PSO-DIC controller for specific trajectory at different power levels.

\section{Competing Interests}

The authors declare that there are no competing interests regarding the publication of this paper.

\section{Acknowledgments}

This research was supported by the Ministry of Science and Technology of Indonesia and National Nuclear Agency of Indonesia. The authors would like to thank them for their support in this important work.

\section{References}

[1] J. H. Pérez-Cruz and A. Poznyak, "Neural control for power ascent of a TRIGA reactor," in Proceedings of the 2008 American Control Conference, pp. 2190-2195, IEEE, Seattle, Wash, USA, 2008.

[2] IAEA, "IAEA safety standards for research reactors," Tech. Rep. SSG-37, 2007.

[3] D. H. Kim, "Nuclear steam generator level control by a neural network-tuning 2-DOF PID controller," in Proceedings of the IEEE International Conference on Computational Intelligence for 
Measurement Systems and Applications (CIMSA '04), pp. 168173, July 2004.

[4] J. H. Perez-Cruz, I. Chairez, A. Poznyak, and J. J. de Rubio, "Constrained neural control for the adaptive tracking of power profiles in a triga reactor," International Journal of Innovative Computing, Information and Control, vol. 7, no. 7, pp. 4575-4788, 2011.

[5] C. Liu, J.-F. Peng, F.-Y. Zhao, and C. Li, "Design and optimization of fuzzy-PID controller for the nuclear reactor power control," Nuclear Engineering and Design, vol. 239, no. 11, pp. 2311-2316, 2009.

[6] Y.-J. Lee and M.-G. Na, "Robust controller design for the nuclear reactor power by extended frequency response method," Nuclear Engineering and Technology, vol. 38, no. 6, pp. 551-560, 2006.

[7] A. Savran, "Multifeedback-layer neural network," IEEE Transactions on Neural Networks, vol. 18, no. 2, pp. 373-384, 2007.

[8] J.-R. Zhang, J. Zhang, T.-M. Lok, and M. R. Lyu, "A hybrid particle swarm optimization-back-propagation algorithm for feedforward neural network training," Applied Mathematics and Computation, vol. 185, no. 2, pp. 1026-1037, 2007.

[9] E. D. Übeyli and M. Übeyli, "Estimation of radiation damage at the structural materials of a hybrid reactor by probabilistic neural networks," Expert Systems with Applications, vol. 36, no. 3, pp. 5184-5189, 2009.

[10] J. A. Carlos Canedo Medeiros and R. Schirru, "Identification of nuclear power plant transients using the Particle Swarm Optimization algorithm," Annals of Nuclear Energy, vol. 35, no. 4, pp. 576-582, 2008.

[11] F. Khoshahval, H. Minuchehr, and A. Zolfaghari, "Performance evaluation of PSO and GA in PWR core loading pattern optimization," Nuclear Engineering and Design, vol. 241, no. 3, pp. 799-808, 2011.

[12] S. Liu and J. Cai, "Studies of fuel loading pattern optimization for a typical pressurized water reactor (PWR) using improved pivot particle swarm method," Annals of Nuclear Energy, vol. 50, pp. 117-125, 2012.

[13] A. A. de Moura Meneses, M. D. Machado, and R. Schirru, "Particle Swarm Optimization applied to the nuclear reload problem of a Pressurized Water Reactor," Progress in Nuclear Energy, vol. 51, no. 2, pp. 319-326, 2009.

[14] S. Carlos, A. Sanchez, S. Martorell, and J.-F. Villanueva, "Particle Swarm Optimization of safety components and systems of nuclear power plants under uncertain maintenance planning," Advances in Engineering Software, vol. 50, no. 1, pp. 12-18, 2012.

[15] BATAN, Multipurpose Reactor G.A. Siwabessy, Safety Analysis Report, vol. 2, 2008.

[16] M. Boroushaki, M. B. Ghofrani, C. Lucas, and M. J. Yazdanpanah, "Identification and control of a nuclear reactor core (VVER) using recurrent neural networks and fuzzy systems," IEEE Transactions on Nuclear Science, vol. 50, no. 2, pp. 159-174, 2003.

[17] H. Akkurt and Ü. Çolak, "PWR system simulation and parameter estimation with neural networks," Annals of Nuclear Energy, vol. 29, no. 17, pp. 2087-2103, 2002.

[18] D. Sutarya and B. Kusumoputro, "Identification of industrial furnace temperature for sintering process in nuclear fuel fabrication using NARX neural networks," Science and Technology of Nuclear Installations, vol. 2014, Article ID 854569, 8 pages, 2014.
[19] R. C. Eberhart and J. Kennedy, "A new optimizer using particle swarm theory," in Proceedings of the 6th International Symposium on Micro Machine and Human Science (MHS '95), pp. 3943, Nagoya, Japan, October 1995.

[20] R. C. Eberhart and Y. Shi, "Particle swarm optimization: developments, applications and resources," in Proceedings of the 2001 Congress on Evolutionary Computation, pp. 81-86, IEEE, Seoul, Republic of Korea, 2001. 

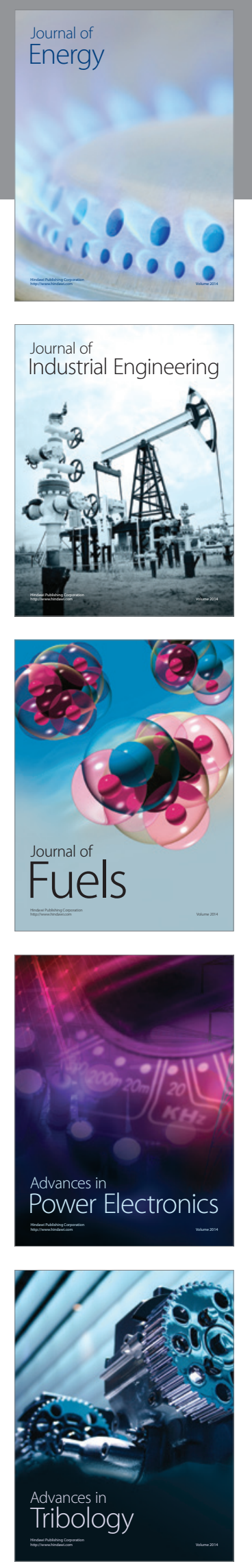
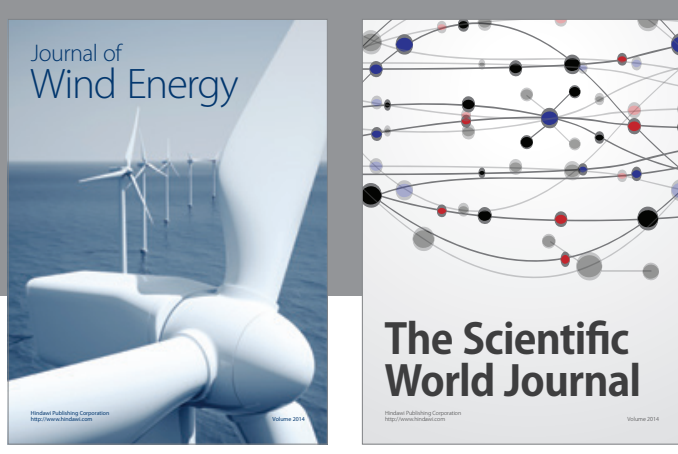

The Scientific World Journal
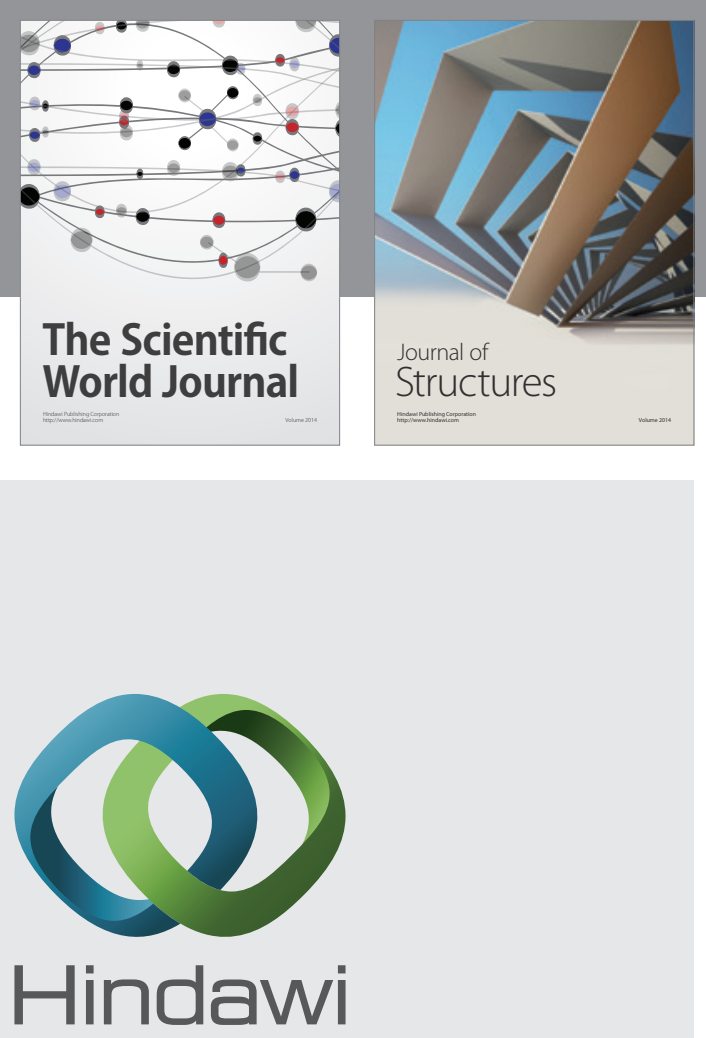

Submit your manuscripts at

http://www.hindawi.com
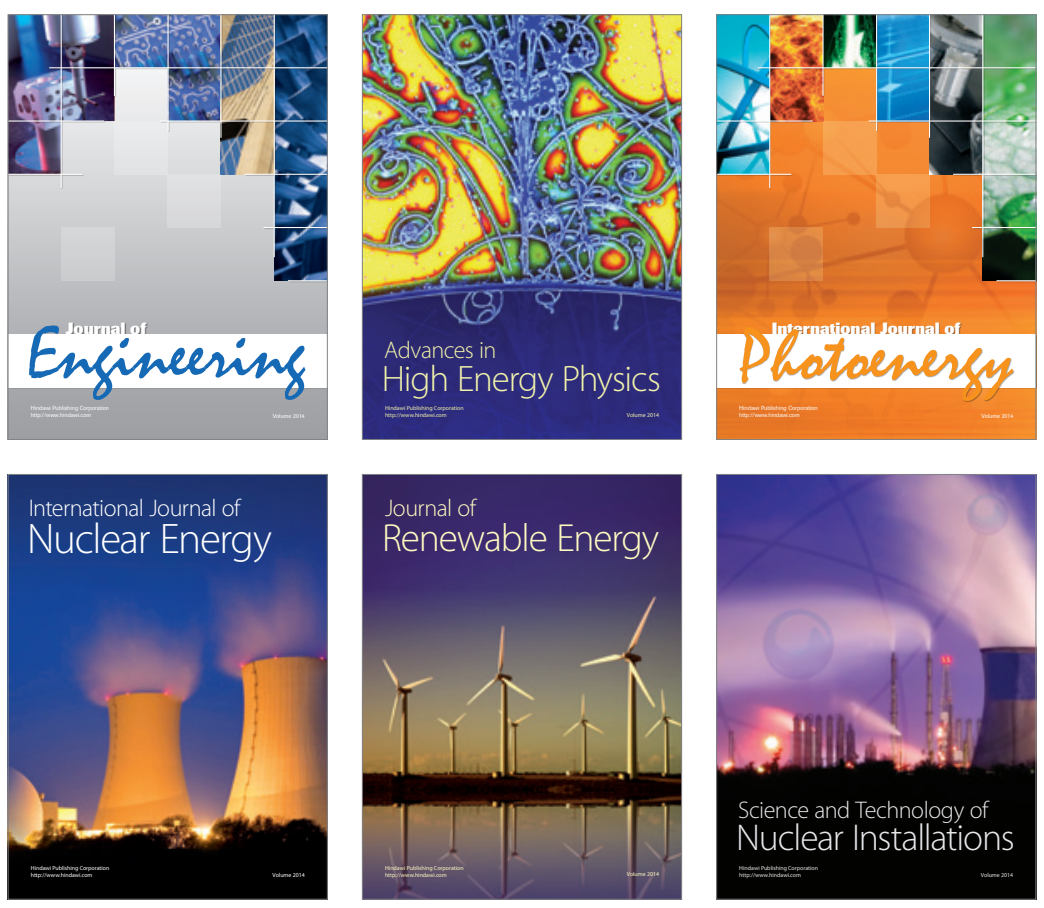
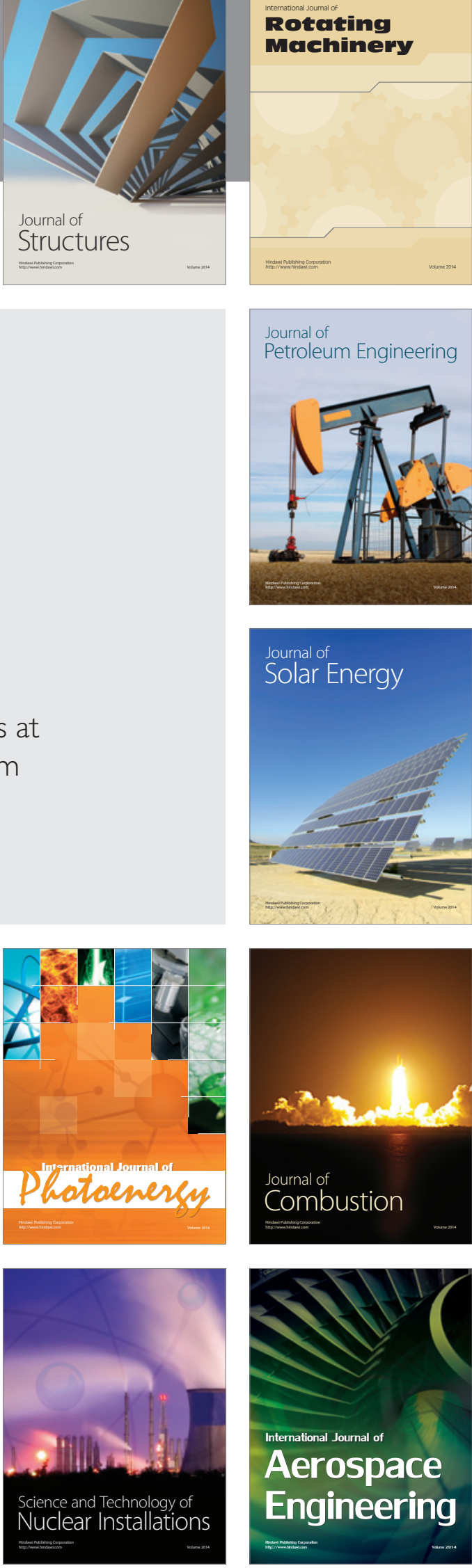